\title{
Algebraic rank on hyperelliptic graphs and graphs of genus 3
}

\author{
Shu Kawaguchi and Kazuhiko Yamaki
}

\begin{abstract}
Let $\bar{G}=(G, \omega)$ be a vertex-weighted graph, and let $\delta$ be a divisor class on $G$. Let $r_{\bar{G}}(\delta)$ denote the (combinatorial) rank of $\delta$. Caporaso has introduced the algebraic $\operatorname{rank} r_{\bar{G}}^{\operatorname{alg}}(\delta)$ of $\delta$ by using nodal curves with dual graph $\bar{G}$. In this paper, when $\bar{G}$ is hyperelliptic or of genus 3 , we show that $r_{\bar{G}}^{\text {alg }}(\delta) \geq r_{\bar{G}}(\delta)$ holds, generalizing our previous result. We also show that, with respect to the specialization map from a nonhyperelliptic curve of genus 3 to its reduction graph, any divisor on the graph lifts to a divisor on the curve of the same rank.
\end{abstract}

\section{Introduction}

Let $k$ be an algebraically closed field. The correspondence between nodal curves over $k$ and their (vertex-weighted) dual graphs appears naturally in algebraic geometry, as in the description of the stratification of the Deligne-Mumford moduli space of stable curves. Recently, a theory of divisors on graphs has been developed (see, e.g., [2], [3], [5], [6]). This enables one to study the relationship between linear systems on a nodal curve and those on the corresponding graph (and also between linear systems on the generic fiber and those on the dual graph of the special fiber of a semistable curve over a discrete valuation ring; see, e.g., [1], [4], [8]-[10], [13], [14]). In particular, a tropical proof of the Brill-Noether theorem has been obtained in [13].

In this development, Caporaso [9] has defined the algebraic rank $r_{\bar{G}}^{\text {alg }}(\delta)$ of a divisor class $\delta$ on a vertex-weighted graph $\bar{G}=(G, \omega)$ by using nodal curves with dual graph $\bar{G}$. It was shown in [9, Summary 3.4$]$ that, on some graphs $\bar{G}$, the algebraic rank $r_{\bar{G}}^{\text {alg }}(\delta)$ equals the combinatorial $\operatorname{rank} r_{\bar{G}}(\delta)$ for any divisor class $\delta$. Further, Caporaso, Len, and Melo [11] have recently shown that $r_{\bar{G}}^{\text {alg }}(\delta) \leq r_{\bar{G}}(\delta)$ holds for any divisor class $\delta$ on any vertex-weighted graph $\bar{G}$. In [14, Proposition 1.5], we have shown that if $\operatorname{char}(k) \neq 2$ and $\bar{G}$ is a hyperelliptic vertex-weighted graph satisfying a certain assumption on the bridges of $G$, then $r_{\bar{G}}^{\text {alg }}(\delta) \geq r_{\bar{G}}(\delta)$ holds for any divisor class $\delta$.

Kyoto Journal of Mathematics, Vol. 56, No. 1 (2016), 177-196 DOI 10.1215/21562261-3445192, (C) 2016 by Kyoto University

Received November 14, 2014. Revised January 16, 2015. Accepted January 19, 2015.

2010 Mathematics Subject Classification: 14H25, 14C20, 14T05, 05 C99.

Kawaguchi's work partially supported by KAKENHI 24740015.

Yamaki's work partially supported by KAKENHI 26800012. 
In this paper, first, we show, based on [14, Proposition 1.5], that the above assumption on the bridges for hyperelliptic graphs is not necessary.

THEOREM 1.1

Assume that $\operatorname{char}(k) \neq 2$. Let $\bar{G}=(G, \omega)$ be a hyperelliptic vertex-weighted graph. Then, for any divisor class $\delta$ on $G$, we have $r_{\bar{G}}^{\text {alg }}(\delta) \geq r_{\bar{G}}(\delta)$.

Second, we show the same inequality on nonhyperelliptic graphs of genus 3 .

\section{THEOREM 1.2}

Let $\bar{G}=(G, \omega)$ be a vertex-weighted graph. Assume that $\bar{G}$ is nonhyperelliptic and of genus 3 . Then, for any divisor class $\delta$ on $G$, we have $r_{\bar{G}}^{\operatorname{alg}}(\delta) \geq r_{\bar{G}}(\delta)$.

These results, combined with the above result of Caporaso, Len, and Melo, show that the algebraic rank equals the combinatorial rank on all hyperelliptic vertexweighted graphs (when $\operatorname{char}(k) \neq 2$ ) and nonhyperelliptic vertex-weighted graphs of genus 3 (and certain graphs which are built from hyperelliptic vertex-weighted graphs and vertex-weighted graphs of genus at most 3; see Remark 5.3).

Caporaso [9, Conjecture 2.1] conjectured that the algebraic rank equals the combinatorial rank on any vertex-weighted graphs. It turns out that this is not the case in general. Caporaso, Len, and Melo [11] have found counterexamples, which we have learned about while preparing this article. Since there are many graphs on which the algebraic rank equals the combinatorial rank (see Remark 5.3), it will be an interesting question to characterize such graphs. See also [12] for very recent progress.

To prove Theorem 1.1, we study the algebraic and combinatorial ranks of vertex-weighted graphs with a bridge.

\section{PROPOSITION 1.3}

Let $\bar{G}=(G, \omega)$ be a vertex-weighted graph having a bridge e with endpoints $v_{1}, v_{2}$. Let $G_{1}$ and $G_{2}$ be the connected components of $G \backslash\{e\}$ such that $v_{1} \in V\left(G_{1}\right)$, $v_{2} \in V\left(G_{2}\right)$, and set $\bar{G}_{i}=\left(G_{i},\left.\omega\right|_{V\left(G_{i}\right)}\right)$ for $i=1,2$. Let $\underline{d} \in \operatorname{Div}(G)$, and let $\underline{d_{i}} \in$ $\operatorname{Div}\left(G_{i}\right)$ be the restriction of $\underline{d}$ to $G_{i}$. Then we have

$$
r_{\bar{G}}(\underline{d}) \leq \begin{cases}r_{\bar{G}_{1}}\left(\underline{d_{1}}\right)+r_{\bar{G}_{2}}\left(\underline{d_{2}}\right)+1 & \text { if } v_{i} \in \mathrm{Bs}\left(\left|\underline{d_{i}}\right|^{\bullet}\right) \text { for each } i=1,2, \\ r_{\bar{G}_{1}}\left(\underline{d_{1}}\right)+r_{\bar{G}_{2}}\left(\underline{d_{2}}\right) & \text { otherwise. }\end{cases}
$$

(For the definition of $\operatorname{Bs}\left(\left|\underline{d_{i}}\right|^{\bullet}\right)$, see Sections 2.1 and 2.2.)

There is a formula corresponding to (1.1) (with the inequality replaced by equality) for nodal curves (see Lemma 3.3). We prove Theorem 1.1 by induction on the number of bridges, using Proposition 1.3, Lemma 3.3, and [14, Corollary 1.7]. (The induction step is a little tricky because of base points (see Lemma 4.1).) 
To prove Theorem 1.2, we show the following proposition (see Section 2.4 for the notation).

PROPOSITION 1.4

Let $R$ be a complete discrete valuation ring with fractional field $\mathbb{K}$ and residue field $k$. Let $\bar{G}=(G, \omega)$ be a nonhyperelliptic graph of genus 3 . Let $\mathscr{X}$ be a regular, generically smooth, semistable $R$-curve with reduction graph $\bar{G}$. Then the following condition (F) holds.

(F) For any $\underline{d} \in \operatorname{Div}(G)$, there exists a divisor $\widetilde{D} \in \operatorname{Div}\left(\mathscr{X}_{\mathbb{K}}\right)$ such that $\widetilde{\rho}_{*}(\widetilde{D})=\underline{d}$ and $r_{\bar{G}}(\underline{d})=r_{\mathscr{X}_{\mathbb{K}}}(\widetilde{D})$,

where $\mathscr{X}_{\mathbb{K}}$ is the generic fiber of $\mathscr{X}$ and $\widetilde{\rho}_{*}: \operatorname{Div}\left(\mathscr{X}_{\mathbb{K}}\right) \rightarrow \operatorname{Div}(G)$ is the specialization map defined in (2.2).

We remark that a similar result for hyperelliptic graphs under a necessary assumption on their bridges is obtained in [14, Theorem 8.2] (see Remark 5.2; see also Proposition 5.1 for a related result, which says that any nonhyperelliptic graph of genus 3 satisfies [14, condition (C)]). The proof of Proposition 1.4 uses the specialization lemma of Amini and Caporaso [2, Theorem 4.10], which is based on Baker's specialization lemma [4, Lemma 2.8], and Raynaud's theorem on the surjectivity of the specialization map between principal divisors (see [16], [4, Corollary A2], Theorem A.1). Then we deduce Theorem 1.2 from Proposition 1.4 by the same argument as that in [14], which is due to Caporaso.

In relation to [11, Question 5.18], the referee has asked whether, also in $\operatorname{char}(k)=2$, the algebraic rank and the combinatorial rank coincide for hyperelliptic graphs. At this moment, we do not know a satisfactory answer.

\section{Combinatorial and algebraic ranks of divisors on graphs}

In this section, we recall definitions and properties of combinatorial and algebraic ranks of divisors on graphs, which will be used later.

\subsection{Divisors on finite graphs}

We briefly recall the theory of divisors on finite graphs. Our basic references are [5] and [6].

Throughout this paper, a finite graph means an unweighted, finite connected graph. We allow a finite graph to have loops and multiple edges. For a finite graph $G$, let $V(G)$ denote the set of vertices, and let $E(G)$ denote the set of edges. The genus of $G$ is defined as $g(G)=|E(G)|-|V(G)|+1$. An edge $e \in E(G)$ is called a bridge if the deletion of $e$ makes $G$ disconnected.

Let $\operatorname{Div}(G)$ be the free abelian group generated by $V(G)$. We call the elements of $\operatorname{Div}(G)$ divisors on $G$. Any divisor $\underline{d} \in \operatorname{Div}(G)$ is uniquely written as $\underline{d}=\sum_{v \in V(G)} n_{v}[v]$ for $n_{v} \in \mathbb{Z}$. The coefficient $n_{v}$ at $[v]$ is denoted by $\underline{d}(v)$. A divisor $\underline{d}$ is effective, written as $\underline{d} \geq 0$, if $\underline{d}(v) \geq 0$ for any $v \in V(G)$. The degree of a divisor $\underline{d}$ is defined as $\operatorname{deg}(\underline{d})=\sum_{v \in V(G)} \underline{d}(v)$. 
A rational function on $G$ is an integer-valued function on $V(G)$. We denote by $\operatorname{Rat}(G)$ the set of rational functions on $G$. For $f \in \operatorname{Rat}(G)$ and a vertex $v$ of $G$, we set $\operatorname{ord}_{v}(f)=\sum_{e=\overline{w v} \in E(G)}(f(w)-f(v))$, where the $e$ 's run through all the edges of $G$ with endpoint $v$. Then

$$
\operatorname{div}(f):=\sum_{v \in V(G)} \operatorname{ord}_{v}(f)[v]
$$

is a divisor on $G$. The set of principal divisors on $G$ is defined as $\operatorname{Prin}(G)=$ $\{\operatorname{div}(f) \mid f \in \operatorname{Rat}(G)\}$. Then $\operatorname{Prin}(G)$ is a subgroup of $\operatorname{Div}(G)$, and we write $\operatorname{Pic}(G)=\operatorname{Div}(G) / \operatorname{Prin}(G)$. For a divisor $\underline{d} \in \operatorname{Div}(G)$, let $\operatorname{cl}(\underline{d})$ denote its divisor class in $\operatorname{Pic}(G)$.

Two divisors $\underline{d}, \underline{d^{\prime}} \in \operatorname{Div}(G)$ are said to be linearly equivalent, expressed as $\underline{d} \sim \underline{d}^{\prime}$, if $\underline{d}-\underline{d}^{\prime} \in \operatorname{Prin}(G)$. For $\underline{d} \in \operatorname{Div}(G)$, the complete linear system $|\underline{d}|$ is defined by

$$
|\underline{d}|=\left\{\underline{d^{\prime}} \in \operatorname{Div}(G) \mid \underline{d^{\prime}} \geq 0, \underline{d^{\prime}} \sim \underline{d}\right\} .
$$

\section{DEFINITION 2.1 ((COMBINATORIAL) RANK OF A DIVISOR [5])}

Let $G$ be a finite graph. Let $\underline{d} \in \operatorname{Div}(G)$. If $|\underline{d}|=\emptyset$, then we set $r_{G}(\underline{d}):=-1$. If $|\underline{d}| \neq \emptyset$, then we set

$$
r_{G}(\underline{d}):=\max \left\{s \in \mathbb{Z}_{\geq 0}|| \underline{d}-\underline{e} \mid \neq \emptyset \text { for any effective divisor } \underline{e} \text { with } \operatorname{deg}(\underline{e})=s\right\} .
$$

We note that $r_{G}(\underline{d})$ depends only on the divisor class of $\underline{d}$. For $\delta=\operatorname{cl}(\underline{d}) \in \operatorname{Pic}(G)$, we set $r_{G}(\delta):=r_{G}(\underline{d})$.

A vertex $v \in V(G)$ is called a base point of the complete linear system $|\underline{d}|$ if $r_{G}(\underline{d}-[v])=r_{G}(\underline{d})$. The set of base points of $|\underline{d}|$ is denoted by $\operatorname{Bs}(|\underline{d}|)$. If $|\underline{d}|=\emptyset$, then any vertex of $G$ is a base point of $|\underline{d}|$ by definition.

In the rest of this Section 2.1, we assume that $G$ is loopless. For any subset $A \subseteq V(G)$ and $v \in V(G)$, the out-degree of $v$ from $A$, $\operatorname{denoted~by~outdeg~}_{A}(v)$, is the number of edges of $G$ having $v$ as one endpoint and whose other endpoint lies in $V(G) \backslash A$. For $\underline{d} \in \operatorname{Div}(G)$, a vertex $v \in A$ is saturated for $\underline{d}$ with respect to $A$ if $\underline{d}(v) \geq \operatorname{outdeg}_{A}(v)$, and nonsaturated otherwise.

\section{DEFINITION 2.2 ( $v_{0}$-REDUCED DIVISOR [5])}

Fix a base vertex $v_{0} \in V(G)$. A divisor $\underline{d} \in \operatorname{Div}(G)$ is called a $v_{0}$-reduced divisor if $\underline{d}(v) \geq 0$ for any $v \in V(G) \backslash\left\{v_{0}\right\}$, and every nonempty subset $A$ of $V(G) \backslash\left\{v_{0}\right\}$ contains a nonsaturated vertex $v \in A$ for $\underline{d}$ with respect to $A$.

We recall from [5] key properties of $v_{0}$-reduced divisors, which will be used later.

\section{PROPOSITION 2.3 ([5, PROPOSITION 3.1 AND ITS PROOF])}

Fix a base vertex $v_{0} \in V(G)$. Then for any $\underline{d} \in \operatorname{Div}(G)$, there exists a unique $v_{0}$-reduced divisor $\underline{d^{\prime}} \in \operatorname{Div}(G)$ that is linearly equivalent to $\underline{d}$. Further, $r_{G}(\underline{d}) \geq 0$ if and only if $\underline{d^{\prime}}$ is effective. 
The canonical divisor $K_{G}$ on $G$ is defined by $K_{G}=\sum_{v \in V(G)}(\operatorname{val}(v)-2)[v] \in$ $\operatorname{Div}(G)$, where $\operatorname{val}(v)$ denotes the number of edges with endpoint $v$. We remark that, with the above definition of rank, the notion of $v_{0}$-reduced divisors, and the canonical divisor on $G$, Baker and Norine [5, Theorem 1.12] established the Riemann-Roch theorem on a loopless finite graph.

Finally, we recall the definition of hyperelliptic graphs.

\section{DEFINITION 2.4 (HYPERELLIPTIC GRAPH [6])}

A loopless finite graph $G$ of $g(G) \geq 2$ is said to be hyperelliptic if there exists a divisor $\underline{d} \in \operatorname{Div}(G)$ such that $\operatorname{deg}(\underline{d})=2$ and $r_{G}(\underline{d})=1$.

\subsection{Rank of divisors on vertex-weighted graphs}

We briefly recall the theory of divisors on vertex-weighted graphs. Our basic references are [2] and [9].

A vertex-weighted graph $\bar{G}=(G, \omega)$ is the pair of a finite graph $G$ and a function (called a vertex-weight function) $\omega: V(G) \rightarrow \mathbb{Z}_{\geq 0}$. The genus of $\bar{G}$ is defined as $g(\bar{G})=g(G)+\sum_{v \in V(G)} \omega(v)$.

For a vertex-weighted graph $\bar{G}=(G, \omega)$, we make a loopless finite graph $\bar{G}^{\bullet}$ as follows. We add $\omega(v)$ loops to $G$ at $v$ for every vertex $v \in V(G)$. Then we insert a vertex in every loop edge. The graph $\bar{G}^{\bullet}$ is called the virtual loopless finite graph of $\bar{G}$. We remark that $G$ may have loops, but $\bar{G}^{\bullet}$ does not.

We have natural embeddings of the vertices $V(G) \subseteq V\left(\bar{G}^{\bullet}\right)$, and of the divisor groups $\operatorname{Div}(G) \subseteq \operatorname{Div}\left(\bar{G}^{\bullet}\right)$. For $\underline{d} \in \operatorname{Div}(G)$, the rank $r_{\bar{G}}(\underline{d})$ of $\underline{d}$ is defined by

$$
r_{\bar{G}}(\underline{d}):=r_{\bar{G}} \bullet(\underline{d}),
$$

where the right-hand side is defined in Definition 2.1. Since $\operatorname{Prin}(G) \subseteq \operatorname{Prin}\left(\bar{G}^{\bullet}\right)$, $r_{\bar{G}}(\underline{d})$ depends only on the divisor class of $\underline{d}$. For $\delta=\operatorname{cl}(\underline{d}) \in \operatorname{Pic}(G)$, we set $r_{\bar{G}}(\delta):=r_{\bar{G}}(\underline{d})$.

For $\underline{d} \in \operatorname{Div}(G)$, we write $|\underline{d}|^{\bullet}$ for the complete linear system $|\underline{d}|$ on $\bar{G}^{\bullet}$. Namely, we have

$$
|\underline{d}|^{\bullet}:=\left\{\underline{d^{\prime}} \in \operatorname{Div}\left(\bar{G}^{\bullet}\right) \mid \underline{d^{\prime}} \geq 0, \underline{d^{\prime}} \text { is linearly equivalent to } \underline{d} \text { in } \bar{G} \bullet .\right.
$$

Here we use the notation $\bullet$ to emphasize that we are considering divisors on $\bar{G}^{\bullet}$.

Let $K_{\bar{G}} \bullet$ be the canonical divisor of $\bar{G}^{\bullet}$. Then the support of $K_{\bar{G}} \bullet$ lies in $V(G)$. We regard $K_{\bar{G}} \bullet$ as an element of $\operatorname{Div}(G)$, and we define the canonical divisor $K_{\bar{G}}$ of $\bar{G}$ by $K_{\bar{G}}:=K_{\bar{G}} \bullet \in \operatorname{Div}(G)$. We remark that if $G$ is loopless, then $K_{\bar{G}}=K_{G}+\sum_{v \in V(G)} 2 \omega(v)[v]$.

A vertex-weighted graph $\bar{G}$ of $g(\bar{G}) \geq 2$ is said to be hyperelliptic if its virtual loopless finite graph $\bar{G}^{\bullet}$ is hyperelliptic. By Definition 2.4, this is equivalent to the existence of $\underline{d} \in \operatorname{Div}\left(\bar{G}^{\bullet}\right)$ such that $\operatorname{deg}(\underline{d})=2$ and $r_{\bar{G}}(\underline{d})=1$.

\subsection{Algebraic rank}

Following [9], we recall the notion of the algebraic rank of a divisor class $\delta$ on a vertex-weighted graph. 
Let $k$ be a fixed algebraically closed field. By a nodal curve, we mean a connected, reduced, projective, one-dimensional scheme over $k$ with at most ordinary double points as singularities.

For a nodal curve $X$, the group of Cartier divisors is denoted by $\operatorname{Div}(X)$. We set $\operatorname{Pic}(X)=\operatorname{Div}(X) / \operatorname{Prin}(X)$, where $\operatorname{Prin}(X)$ denotes the group of principal divisors. For $L \in \operatorname{Pic}(X)$, we write $r_{X}(L)=\operatorname{dim}_{k} H^{0}(X, L)-1$.

Given a nodal curve $X$, the (vertex-weighted) dual graph $\bar{G}=(G, \omega)$ associated to $X$ is defined as follows. Let $C_{1}, \ldots, C_{r}$ be the irreducible components of $X$. Then $G$ has vertices $v_{1}, \ldots, v_{r}$ which correspond to $C_{1}, \ldots, C_{r}$, respectively. Two vertices $v_{i}, v_{j}(i \neq j)$ of $G$ are connected by $a_{i j}$ edges if $\# C_{i} \cap C_{j}=a_{i j}$. A vertex $v_{i}$ has $b_{i}$ loops if \# $\operatorname{Sing}\left(C_{i}\right)=b_{i}$. The vertex-weighted function $\omega$ is given by assigning to $v_{i}$ the geometric genus of $X_{i}$.

Let $\bar{G}=(G, \omega)$ be a vertex-weighted graph. Let $M^{\text {alg }}(\bar{G})$ be a family of nodal curves representing all the isomorphism classes of nodal curves with dual graph $\bar{G}$. For $X \in M^{\text {alg }}(\bar{G})$, we write $X=\bigcup_{v \in V(G)} C_{v}$, where $C_{v}$ is the irreducible curve corresponding to $v \in V(G)$. We have a natural map

$$
\rho_{*}: \operatorname{Div}(X) \rightarrow \operatorname{Div}(G), \quad D \mapsto \sum_{v \in V(G)}\left(\operatorname{deg}\left(\left.D\right|_{C_{v}}\right)\right)[v] .
$$

In other words, for a Cartier divisor $D$ on $X, \rho_{*}(D) \in \operatorname{Div}(G)$ gives the multidegree of $D$. Since linear equivalent divisors on $X$ have the same multidegree, $\rho_{*}$ descends to $\operatorname{Pic}(X) \rightarrow \operatorname{Div}(G)$. Then we have a stratification of $\operatorname{Pic}(X)$ :

$$
\operatorname{Pic}(X)=\bigsqcup_{\underline{d} \in \operatorname{Div}(G)} \operatorname{Pic}^{\underline{d}}(X),
$$

where $\operatorname{Pic}^{\underline{d}}(X)=\left\{L \in \operatorname{Pic}(X) \mid \operatorname{deg}\left(\left.L\right|_{C_{v}}\right)=d_{v}\right.$ for any $\left.v \in V(G)\right\}$ for $\underline{d}=$ $\left(d_{v}\right)_{v \in V(G)} \in \operatorname{Div}(G)$.

\section{DEFINITION 2.5 (ALGEBRAIC RANK [9])}

Let $\bar{G}=(G, \omega)$ be a vertex-weighted graph, and let $\delta \in \operatorname{Pic}(G)$ be a divisor class on $G$. We set

$$
r_{\bar{G}}^{\operatorname{alg}}(\delta)=\max _{X \in M^{\operatorname{alg}}(\bar{G})}\left\{\min _{\underline{d} \in \delta}\left\{\max _{L \in \operatorname{Pic}^{\underline{d}}(X)}\left\{r_{X}(L)\right\}\right\}\right\},
$$

and call $r_{\bar{G}}^{\mathrm{alg}}(\delta)$ the algebraic rank of the divisor class $\delta$.

\subsection{The specialization lemma for vertex-weighted graphs}

We recall the specialization lemma for vertex-weighted graphs due to Amini and Caporaso [2], which generalizes Baker's specialization lemma [4, Lemma 2.8] for loopless finite graphs. Our basic references are [2] and [4].

Let $k$ be a fixed algebraically closed field. Let $R$ be a complete discrete valuation ring with residue field $k$. Let $\mathbb{K}$ denote the fractional field of $R$.

By an $R$-curve, we mean an integral scheme of dimension 2 that is proper and flat over $\operatorname{Spec}(R)$. For an $R$-curve $\mathscr{X}$, we denote by $\mathscr{X}_{\mathbb{K}}$ the generic fiber of $\mathscr{X}$, and by $X$ the special fiber of $\mathscr{X}$. We say that $\mathscr{X}$ is a semistable $R$-curve 
if $X$ is a nodal curve. The vertex-weighted dual graph $\bar{G}=(G, \omega)$ of $X$ is then called the reduction graph of $\mathscr{X}$.

Let $\mathscr{X}$ be a regular, generically smooth, semistable $R$-curve. Since $\mathscr{X}_{\mathbb{K}}$ is smooth (resp., $\mathscr{X}$ is regular), the group of Cartier divisors on $\mathscr{X}_{\mathbb{K}}$ (resp., $\mathscr{X}$ ) is the same as the group of Weil divisors. The Zariski closure of an effective divisor on $\mathscr{X}_{\mathbb{K}}$ in $\mathscr{X}$ is a Cartier divisor. Extending by linearity, one can associate to any divisor on $\mathscr{X}_{\mathbb{K}}$ a Cartier divisor on $\mathscr{X}$, which is also called the Zariski closure of the divisor.

Let $\widetilde{D}$ be a divisor on $\mathscr{X}_{\mathbb{K}}$, and let $\widetilde{\mathscr{D}}$ be the Zariski closure of $\widetilde{D}$. Let $\mathcal{O}_{\mathscr{X}}(\widetilde{\mathscr{D}})$ be the invertible sheaf on $\mathscr{X}$ associated to $\widetilde{\mathscr{D}}$. We define the specialization map $\widetilde{\rho}_{*}: \operatorname{Div}\left(\mathscr{X}_{\mathbb{K}}\right) \rightarrow \operatorname{Div}(G)$ by (see [4, Section 2.1])

$$
\widetilde{\rho}_{*}(\widetilde{D}):=\sum_{v \in V(G)} \operatorname{deg}\left(\left.\mathcal{O}_{\mathscr{X}}(\widetilde{\mathscr{D}})\right|_{C_{v}}\right)[v] \in \operatorname{Div}(G)
$$

The map $\widetilde{\rho}_{*}$ is compatible with the map $\rho_{*}$ in (2.1). Namely, let $D \in \operatorname{Div}(X)$ be a Cartier divisor on the special fiber such that the associated invertible sheaf $\mathcal{O}_{X}(D)$ is isomorphic to $\left.\mathcal{O}_{\mathscr{X}}(\widetilde{\mathscr{D}})\right|_{X}$. Then, by definition, we have

$$
\rho_{*}(D)=\widetilde{\rho}_{*}(\widetilde{D}) .
$$

\section{REMARK 2.6}

In [14], $\widetilde{\rho}_{*}$ is denoted by $\rho_{*}$. Here we use the notation $\widetilde{\rho}_{*}$, for we have already used the notation $\rho_{*}$ in $(2.1)$.

Let $\operatorname{Div}(\mathscr{X}(\mathbb{K}))$ be the subgroup of $\operatorname{Div}\left(\mathscr{X}_{\mathbb{K}}\right)$ generated by $\mathbb{K}$-valued points of $\mathscr{X}$. Then

$$
\left.\widetilde{\rho}_{*}\right|_{\operatorname{Div}\left(\mathscr{X}_{\mathbb{K}}\right)}: \operatorname{Div}(\mathscr{X}(\mathbb{K})) \rightarrow \operatorname{Div}(G)
$$

is surjective (see [4, Remark 2.3], [15, Proposition 10.1.40(b)]).

THEOREM 2.7 (AMINI-CAPORASO'S SPECIALIZATION LEMMA [2, THEOREM 4.10])

Let $\mathscr{X}$ be a regular, generically smooth, semistable $R$-curve with reduction graph $\bar{G}=(G, \omega)$. Then, for any $\widetilde{D} \in \operatorname{Div}\left(\mathscr{X}_{\mathbb{K}}\right)$, one has $r_{\bar{G}}\left(\widetilde{\rho}_{*}(\widetilde{D})\right) \geq r_{\mathscr{X}_{\mathbb{K}}}(\widetilde{D})$.

Theorem 2.7 is a generalization of Baker's specialization lemma [4, Lemma 2.8] for loopless finite graphs. Although Amini and Caporaso consider a smooth quasiprojective curve $B$ over $k$ (in place of $\operatorname{Spec}(R)$ ), that is, they consider a morphism $\phi: \mathscr{X} \rightarrow B$, we remark that their arguments also work over $\operatorname{Spec}(R)$. (By the surjectivity of the map (2.4), the argument over $R$ works the same as the argument for $\phi: \mathscr{X} \rightarrow B$ that admits a section passing through any given component of the special fiber.)

\section{Reduced divisors and decomposition of graphs}

In this section, we prove Proposition 1.3. We first show some properties of divisors on a graph with a bridge. 
LEMMA 3.1

Let $G$ be a loopless finite graph with a bridge e having endpoints $v_{1}, v_{2}$. Let $G_{1}$ and $G_{2}$ be the connected components of $G \backslash\{e\}$ such that $v_{1} \in V\left(G_{1}\right), v_{2} \in V\left(G_{2}\right)$. For $i=1,2$, let $\jmath_{i}: V\left(G_{i}\right) \hookrightarrow V(G)$ be the natural embedding, and let $\jmath_{i *}: \operatorname{Div}\left(G_{i}\right) \hookrightarrow$ $\operatorname{Div}(G)$ be the induced map.

(a) For $i=1,2$, we have $\jmath_{i *}\left(\operatorname{Prin}\left(G_{i}\right)\right) \subseteq \operatorname{Prin}(G)$.

(b) For $i=1,2$, let $\underline{d_{i}}$ be a $v_{i}$-reduced divisor on $G_{i}$. Then

$$
\jmath_{1 *}\left(\underline{d_{1}}-\underline{d_{1}}\left(v_{1}\right)\left[v_{1}\right]\right)+\jmath_{2 *}\left(\underline{d_{2}}-\underline{d_{2}}\left(v_{2}\right)\left[v_{2}\right]\right)+\left(\underline{d_{1}}\left(v_{1}\right)+\underline{d_{2}}\left(v_{2}\right)\right)\left[v_{1}\right]
$$

is a $v_{1}$-reduced divisor on $G$.

Proof

(a) We may assume that $i=1$. Let $f_{1}$ be a rational function on $G_{1}$. We extend $f_{1}$ to a rational function $\widetilde{f}_{1}$ on $G$ by setting $\widetilde{f}_{1}(w)=f_{1}\left(v_{1}\right)$ for any $w \in V\left(G_{2}\right)$. Then we have $\operatorname{div}(\widetilde{f})=j_{1 *}\left(\operatorname{div}\left(f_{1}\right)\right)$. Thus, $j_{1 *}\left(\operatorname{div}\left(f_{1}\right)\right) \in \operatorname{Prin}(G)$, which gives the assertion.

(b) We put

$$
\underline{d}:=\jmath_{1 *}\left(\underline{d_{1}}-\underline{d_{1}}\left(v_{1}\right)\left[v_{1}\right]\right)+\jmath_{2 *}\left(\underline{d_{2}}-\underline{d_{2}}\left(v_{2}\right)\left[v_{2}\right]\right) .
$$

Since being $v_{1}$-reduced does not depend on the coefficient of the divisor at $\left[v_{1}\right]$, it suffices to show that $\underline{d}$ is a $v_{1}$-reduced divisor on $G$. Let $A \subseteq V(G) \backslash\left\{v_{1}\right\}$ be any nonempty subset, and we are going to show that there exists a nonsaturated vertex $v \in A$ for $\underline{d}$ with respect to $A$.

If $v_{2} \in A$, then it follows from $v_{1} \notin A$ that outdeg ${ }_{A}\left(v_{2}\right) \geq 1$ (from the contribution of the bridge $e$ ). Since $\underline{d}\left(v_{2}\right)=0$, we see that $v_{2} \in V(G) \backslash\left\{v_{1}\right\}$ is a nonsaturated vertex for $\underline{d}$ with respect to $A$. Thus, we may and do assume that $v_{2} \notin A$, and hence, $A \subseteq V(G) \backslash\left\{v_{1}, v_{2}\right\}$.

We set $A_{1}:=A \cap V\left(G_{1}\right)$ and $A_{2}:=A \cap V\left(G_{2}\right)$. Then $A_{1} \subseteq V(G) \backslash\left\{v_{1}\right\}$ and $A_{2} \subseteq V(G) \backslash\left\{v_{2}\right\}$. Since $A \neq \emptyset$, we have $A_{1} \neq \emptyset$ or $A_{2} \neq \emptyset$. Without loss of generality, we assume that $A_{1} \neq \emptyset$. Since $\underline{d_{1}}$ is a $v_{1}$-reduced divisor on $G_{1}$, there exists a nonsaturated vertex $v \in A_{1}$ for $\underline{d_{1}}$ with respect to $A_{1}$, that is, $\underline{d_{1}}(v)<\operatorname{outdeg}_{A_{1}}(v)$. Since $\underline{d_{1}}(v)=\underline{d}(v)$ and $\operatorname{outdeg}_{A_{1}}(v)=\operatorname{outdeg}_{A}(v)$, we have $\underline{d}(v)<\operatorname{outdeg}_{A}(v)$. Thus, $v \in A$ is a nonsaturated vertex for $\underline{d}$ with respect to $A$, which shows the lemma.

The next lemma will be used in Section 5 .

\section{LEMMA 3.2}

Let $\bar{G}=(G, \omega)$ be a vertex-weighted graph. Let $\underline{d} \in \operatorname{Div}(G)$. If $r_{\bar{G}}(\underline{d}) \geq 0$, then there exists an effective divisor $\underline{e} \in \operatorname{Div}(G)$ that is linearly equivalent to $\underline{d}$ in $G$.

\section{Proof}

Let $\bar{G}^{\bullet}$ be the virtual loopless finite graph of $\bar{G}$. Via the natural embedding of the sets of vertices, we regard $V(G) \subseteq V\left(\bar{G}^{\bullet}\right)$. The condition $r_{\bar{G}}(\underline{d}):=r_{\bar{G}} \bullet(\underline{d}) \geq 0$ 
means that there exists a rational function $\bar{f} \in \operatorname{Rat}\left(\bar{G}^{\bullet}\right)$ such that $\underline{d^{\prime}}:=\underline{d}+\operatorname{div}(\bar{f})$ is an effective divisor on $\bar{G}^{\bullet}$.

Let $w \in V\left(\bar{G}^{\bullet}\right) \backslash V(G)$. This means that $w$ is a vertex inserted in a loop edge. Thus, there exist exactly two edges $e_{1}, e_{2}$ of $\bar{G}^{\bullet}$ with endpoint $w$, and the other endpoint of $e_{1}$ and that of $e_{2}$ are the same, which we denote by $w^{\prime}$. Since $\underline{d}(w)=0$ and $\underline{d^{\prime}}(w) \geq 0$, we see that $\bar{f}\left(w^{\prime}\right) \geq \bar{f}(w)$.

We set $f:=\left.\bar{f}\right|_{V(G)} \in \operatorname{Rat}(G)$. Since $\underline{d} \in \operatorname{Div}(G)$ and $\bar{f}\left(w^{\prime}\right) \geq \bar{f}(w)$ for every $w \in V\left(\bar{G}^{\bullet}\right) \backslash V(G)$, we see that $\underline{e}:=\underline{d}+\operatorname{div}(f)$ is an effective divisor on $G$. This shows the lemma.

We begin the proof of Proposition 1.3.

\section{Proof of Proposition 1.3}

Let $\bar{G}^{\bullet}, \bar{G}_{1}^{\bullet}$, and $\bar{G}_{2}^{\bullet}$ be the virtual loopless finite graphs of $\bar{G}, \bar{G}_{1}$, and $\bar{G}_{2}$, respectively. Note that $\bar{G}^{\bullet}$ is the graph obtained by connecting $\bar{G}_{1}^{\bullet}$ and $\bar{G}_{2}^{\bullet}$ with the edge $e$. For $i=1,2$, let $\jmath_{i *}^{\bullet}: \operatorname{Div}\left(\bar{G}_{i}^{\bullet}\right) \hookrightarrow \operatorname{Div}\left(\bar{G}^{\bullet}\right)$ be the induced embedding of divisors.

Via the natural embedding of the sets of vertices, we regard $V\left(G_{i}\right) \subseteq V(G) \subseteq$ $V\left(\bar{G}^{\bullet}\right)$ and $V\left(G_{i}\right) \subseteq V\left(\bar{G}_{i}^{\bullet} \subseteq \subseteq\left(\bar{G}^{\bullet}\right)\right.$ for $i=1,2$. Thus, in the following argument, we will often identify the vertex $v_{i} \in V\left(G_{i}\right)$ with the corresponding vertices in $G, \bar{G}_{i}^{\bullet}$, and $\bar{G}^{\bullet}$.

For $i=1,2$, we set $r_{i}=r_{\bar{G}_{i}}\left(\underline{d_{i}}\right)$. By the definition of the rank, there exists an effective divisor $\underline{e_{i}} \in \operatorname{Div}\left(G_{i}^{\bullet}\right)$ with $\operatorname{deg}\left(\underline{e_{i}}\right)=r_{i}+1$ such that $r_{\bar{G}_{i}}\left(\underline{d}_{i}-\underline{e_{i}}\right)=$ -1 . We set $\underline{c_{i}}=\underline{d_{i}}-\underline{e_{i}}$, and let $c_{i}^{\prime} \in \operatorname{Div}\left(G_{i}^{\bullet}\right)$ be the $v_{i}$-reduced divisor that is linearly equivalent to $\underline{c_{i}}$ on $G_{i}^{\bullet}$. Since $r_{\bar{G}_{i}}\left(\underline{d_{i}}-\underline{e_{i}}\right)=-1$, we have $\underline{c_{i}^{\prime}}\left(v_{i}\right)<0$ by Proposition 2.3.

We claim that $\left.r_{\bar{G}} \bullet \underline{d}-\jmath_{1 *}^{\bullet}\left(\underline{e_{1}}\right)-\jmath_{2 *}^{\bullet}\left(\underline{e_{2}}\right)\right)=-1$. Indeed, we see from Lemma 3.1(a) that, as divisors on $\overline{\bar{G}^{\bullet}}$,

$$
\begin{aligned}
\underline{d} & -\jmath_{1 *}^{\bullet}\left(\underline{e_{1}}\right)-j_{2 *}^{\bullet}\left(\underline{e_{2}}\right) \\
& =\jmath_{1 *}^{\bullet}\left(\underline{d_{1}}-\underline{e_{1}}\right)+\jmath_{2 *}^{\bullet}\left(\underline{d_{2}}-\underline{e_{2}}\right) \\
& \sim \jmath_{1 *}^{\bullet}\left(\underline{c_{1}^{\prime}}\right)+\jmath_{2 *}^{\bullet}\left(\underline{c_{2}^{\prime}}\right) \\
& \sim \jmath_{1 *}^{\bullet}\left(\underline{c_{1}^{\prime}}-\underline{c_{1}^{\prime}}\left(v_{1}\right)\left[v_{1}\right]\right)+\jmath_{2 *}^{\bullet}\left(\underline{c_{2}^{\prime}}-\underline{c_{2}^{\prime}}\left(v_{2}\right)\left[v_{2}\right]\right)+\left(\underline{c_{1}^{\prime}}\left(v_{1}\right)+\underline{c_{2}^{\prime}}\left(v_{2}\right)\right)\left[v_{1}\right] .
\end{aligned}
$$

We denote by $g$ the divisor in the last line in the above equation. Then, by Lemma 3.1(b), $\underline{g}$ is a $v_{1}$-reduced divisor on $\bar{G} \bullet$ Since $\underline{g}\left(v_{1}\right)=c_{1}^{\prime}\left(v_{1}\right)+c_{2}^{\prime}\left(v_{2}\right)<0$, we have $r_{\bar{G}} \bullet\left(\underline{d}-j_{1 *}^{\bullet}\left(\underline{e_{1}}\right)-j_{2 *}^{\bullet}\left(\underline{e_{2}}\right)\right)=-1$ by Proposition 2.3 .

It follows that

$$
\begin{aligned}
r_{\bar{G}}(\underline{d}) & =r_{\bar{G}} \bullet(\underline{d}) \leq \operatorname{deg}\left(\jmath_{1 *}^{\bullet}\left(\underline{e_{1}}\right)+\jmath_{2 *}\left(\underline{e_{2}}\right)\right)-1 \\
& =r_{1}+r_{2}+1=r_{\bar{G}_{1}}\left(\underline{d_{1}}\right)+r_{\bar{G}_{2}}\left(\underline{d_{2}}\right)+1 .
\end{aligned}
$$

In particular, this inequality shows the first case of the inequality in (1.1). 
Suppose now that $v_{1} \notin \operatorname{Bs}\left(\left|\underline{d}_{1}\right|^{\bullet}\right)$ or $v_{2} \notin \operatorname{Bs}\left(\left|\underline{d}_{2}\right|^{\bullet}\right)$. We need to show the stronger inequality

$$
r_{\bar{G}}(\underline{d}) \leq r_{\bar{G}_{1}}\left(\underline{d_{1}}\right)+r_{\bar{G}_{2}}\left(\underline{d_{2}}\right) .
$$

Without loss of generality, we may assume that $v_{2} \notin \operatorname{Bs}\left(\left|\underline{d}_{2}\right|^{\bullet}\right)$. This means that $\left|\underline{d}_{2}\right|^{\bullet} \neq \emptyset$ and $r_{\bar{G}_{2}}\left(\underline{d_{2}}-\left[v_{2}\right]\right)=r_{2}-1$. In particular, $r_{2} \geq 0$.

By the definition of the rank, there exists an effective divisor $\underline{\widetilde{e}}_{2} \in \operatorname{Div}\left(\bar{G}_{2}^{\bullet}\right)$ with $\operatorname{deg}\left(\underline{\widetilde{e}_{2}}\right)=r_{2}$ such that $r_{\bar{G}_{2}^{\bullet}}\left(\underline{d_{2}}-\left[v_{2}\right]-\underline{\widetilde{e}_{2}}\right)=-1$. We set $\underline{h_{2}}=\underline{\bar{d}_{2}}-\underline{\widetilde{e}_{2}}$, and let $h_{2}^{\prime} \in \operatorname{Div}\left(\bar{G}_{2}^{\bullet}\right)$ be the $v_{2}$-reduced divisor that is linearly equivalent to $\underline{h_{2}}$ on $\bar{G}_{2}^{\bullet}$. Since $r_{\bar{G}_{2}}\left(\underline{d_{2}}\right)=r_{2}$, we have $r_{\bar{G}_{2}^{\bullet}}\left(\underline{d_{2}}-\underline{\widetilde{e}_{2}}\right) \geq 0$; hence, $\underline{h_{2}^{\prime}}$ is an effective divisor on $\bar{G}_{2}^{\bullet}$ by Proposition 2.3. Since $\underline{h_{2}^{\prime}}$ is $v_{2}$-reduced and since $r_{\bar{G}_{2}^{\bullet}}\left(\underline{h_{2}^{\prime}}-\left[v_{2}\right]\right)=$ $r_{\bar{G}_{2}}\left(\underline{d_{2}}-\left[v_{2}\right]-\underline{\tilde{e}_{2}}\right)=-1$, we see that $\underline{h_{2}^{\prime}}\left(v_{2}\right)=0$.

It follows from Lemma 3.1(a) that

$$
\begin{aligned}
\underline{d}-j_{1 *}^{\bullet}\left(\underline{e_{1}}\right)-j_{2 *}^{\bullet}\left(\widetilde{e}_{2}\right) & =j_{1 *}^{\bullet}\left(\underline{d_{1}}-\underline{e_{1}}\right)+j_{2 *}^{\bullet}\left(\underline{d_{2}}-\underline{\tilde{e}_{2}}\right) \\
& \sim j_{1 *}^{\bullet}\left(\underline{c_{1}^{\prime}}\right)+j_{2 *}\left(\underline{h_{2}^{\prime}}\right) .
\end{aligned}
$$

Since $\underline{h_{2}^{\prime}}\left(v_{2}\right)=0$, we see that $\jmath_{1 *}^{\bullet}\left(c_{1}^{\prime}\right)+\jmath_{2 *}^{\bullet}\left(\underline{h_{2}^{\prime}}\right)$ is a $v_{1}$-reduced divisor on $\bar{G}^{\bullet}$ by Lemma 3.1(b). Since $\jmath_{1 *}^{\bullet}\left(\underline{c_{1}^{\prime}}\right)\left(v_{1}\right)+\jmath_{2 *}^{\bullet}\left(h_{2}^{\prime}\right)\left(\overline{\left.v_{1}\right)}=\underline{c_{1}^{\prime}}\left(v_{1}\right)<0\right.$, Proposition 2.3 tells us that $r_{\bar{G}} \bullet\left(\underline{d}-\jmath_{1 *}^{\bullet}\left(\underline{e_{1}}\right)-\overline{j_{2 *}^{\bullet}}\left(\widetilde{e}_{2}\right)\right)=-1$. Then

$$
\begin{aligned}
r_{\bar{G}}(\underline{d}) & =r_{\bar{G}} \bullet(\underline{d}) \leq \operatorname{deg}\left(\jmath_{1 *}\left(\underline{e_{1}}\right)+\jmath_{2 *}^{\bullet}\left(\widetilde{e}_{2}\right)\right)-1 \\
& \left.=r_{1}+r_{2}=r_{\bar{G}_{1}}\left(\underline{d_{1}}\right)+r_{\bar{G}_{2}} \underline{d_{2}}\right) .
\end{aligned}
$$

Thus, we obtain the inequality in the remaining case.

There exists a formula corresponding to Proposition 1.3 (with the inequality replaced by equality) for nodal curves.

\section{LEMMA 3.3}

Let $X$ be a nodal curve. We assume that $X$ has a decomposition as $X=X_{1} \cup X_{2}$ into two nodal curves so that $X_{1}$ and $X_{2}$ meet at exactly one point $p$. Let $D$ be a Cartier divisor on $X$, and we set $D_{i}=\left.D\right|_{X_{i}} \in \operatorname{Div}\left(X_{i}\right)$ for $i=1,2$. Then

$$
r_{X}(D)= \begin{cases}r_{X_{1}}\left(D_{1}\right)+r_{X_{2}}\left(D_{2}\right)+1 & \text { if } p \in \operatorname{Bs}\left(\left|D_{i}\right|\right) \text { for each } i=1,2 \\ r_{X_{1}}\left(D_{1}\right)+r_{X_{2}}\left(D_{2}\right) & \text { otherwise }\end{cases}
$$

Proof

This is a well-known fact, so we omit a proof (see also [9, Remark 1.5]).

The following simple remark will be used in the next section.

\section{REMARK 3.4}

Let $X, X_{1}, X_{2}, p$ be as in Lemma 3.3. For $i=1,2$, let $D_{i}$ be a Cartier divisor on $X_{i}$. Then there exists a Cartier divisor $D$ on $X$ such that $\left.D\right|_{X_{i}}$ is linearly equivalent to $D_{i}$. Indeed, let $p_{i}: X \rightarrow X_{i}$ be the morphism given by the identity 
on $X_{i}$ and the constant map to $p$ on the other component. Let $\mathcal{O}_{X_{i}}\left(D_{i}\right)$ be the invertible sheaf on $X_{i}$ associated to $D_{i}$. Then it suffices to take $D \in \operatorname{Div}(X)$ such that the associated invertible sheaf $\mathcal{O}_{X}(D)$ is isomorphic to $p_{1}^{*}\left(\mathcal{O}_{X_{1}}\left(D_{1}\right)\right) \otimes$ $p_{2}^{*}\left(\mathcal{O}_{X_{2}}\left(D_{2}\right)\right)$.

\section{Graphs with a bridge and hyperelliptic graphs}

In this section, we prove Theorem 1.1. We begin by showing the following lemma.

\section{LEMMA 4.1}

Let $\bar{G}=(G, \omega)$ be a vertex-weighted graph with a bridge e with endpoints $v_{1}, v_{2}$. Let $G_{1}$ and $G_{2}$ be the connected components of $G \backslash\{e\}$ such that $v_{1} \in V\left(G_{1}\right)$ and $v_{2} \in V\left(G_{2}\right)$, and we set $\bar{G}_{i}=\left(G_{i},\left.\omega\right|_{V\left(G_{i}\right)}\right)$ for $i=1,2$. Let $\underline{d} \in \operatorname{Div}(G)$, and let $\underline{d_{i}} \in \operatorname{Div}\left(G_{i}\right)$ be the restriction of $\underline{d}$ to $G_{i}$. Let $X$ be a nodal curve over $k$ with dual graph $\bar{G}$, and we write $X_{i}$ for the union of irreducible components of $X$ corresponding to $\bar{G}_{i}$. Let $\rho_{*}: \operatorname{Div}(X) \rightarrow \operatorname{Div}(G)$ and $\rho_{i *}: \operatorname{Div}\left(X_{i}\right) \rightarrow \operatorname{Div}\left(G_{i}\right)$ be the maps defined in (2.1). For $i=1,2$, we assume that, for any divisor $\underline{e_{i}} \in \operatorname{Div}\left(G_{i}\right)$, there exists a Cartier divisor $E_{i}$ on $X_{i}$ satisfying $\rho_{i *}\left(E_{i}\right)=\underline{e_{i}}$ and $r_{X_{i}}\left(E_{i}\right) \geq$ $r_{\bar{G}_{i}}\left(\underline{e}_{i}\right)$. Then there exists a Cartier divisor $D$ on $X$ satisfying $\rho_{*}(D)=\underline{d}$ and

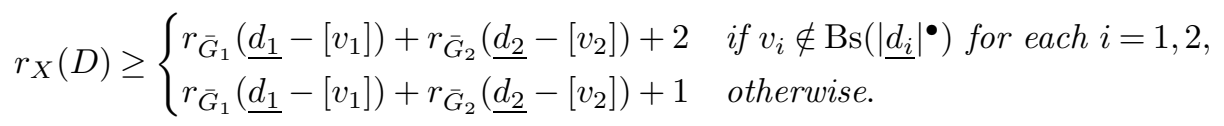

Proof

Case 1. Suppose that $v_{i} \notin \operatorname{Bs}\left(\left|\underline{d}_{i}\right|^{\bullet}\right)$ for each $i=1,2$. This means that $\left|\underline{d_{i}}\right|^{\bullet} \neq \emptyset$ and

$$
r_{\bar{G}_{i}}\left(\underline{d_{i}}-\left[v_{i}\right]\right)=r_{\bar{G}_{i}}\left(\underline{d_{i}}\right)-1 .
$$

We take a Cartier divisor $D_{i}$ on $X_{i}$ satisfying $\rho_{i *}\left(D_{i}\right)=\underline{d_{i}}$ and $r_{X_{i}}\left(D_{i}\right) \geq r_{\bar{G}_{i}}\left(\underline{d_{i}}\right)$. By Remark 3.4, there exists a Cartier divisor $D$ on $X$ such that $\left.D\right|_{X_{i}}$ is linearly equivalent to $D_{i}$ on $X_{i}$. Then we have

$$
\begin{aligned}
\rho_{*}(D) & =\rho_{1 *}\left(\left.D\right|_{X_{1}}\right)+\rho_{2 *}\left(\left.D\right|_{X_{2}}\right) \\
& =\rho_{1 *}\left(D_{1}\right)+\rho_{2 *}\left(D_{2}\right)=\underline{d_{1}}+\underline{d_{2}}=\underline{d} .
\end{aligned}
$$

Further, we have

$$
\begin{aligned}
r_{X}(D) & \geq r_{X_{1}}\left(\left.D\right|_{X_{1}}\right)+r_{X_{2}}\left(\left.D\right|_{X_{2}}\right) \quad(\text { from Lemma 3.3) } \\
& =r_{X_{1}}\left(D_{1}\right)+r_{X_{2}}\left(D_{2}\right) \quad\left(\text { since }\left.D\right|_{X_{i}} \sim D_{i} \text { for each } i=1,2\right) \\
& \left.\geq r_{\bar{G}_{1}} \underline{d_{1}}\right)+r_{\bar{G}_{2}}\left(\underline{d_{2}}\right) \quad\left(\text { from the assumptions on } D_{i}\right) \\
& =r_{\bar{G}_{1}}\left(\underline{d_{1}}-\left[v_{1}\right]\right)+r_{\bar{G}_{2}}\left(\underline{d_{2}}-\left[v_{2}\right]\right)+2 \quad(\text { from }(4.1)) .
\end{aligned}
$$

This gives the desired properties in this case.

Case 2. Suppose that $v_{1} \in \operatorname{Bs}\left(\left|\underline{d_{1}}\right|^{\bullet}\right)$ or $v_{2} \in \operatorname{Bs}\left(\left|\underline{d_{2}}\right|^{\bullet}\right)$. For $i=1$, 2, we take a Cartier divisor $D_{i}^{\prime}$ on $X_{i}$ satisfying $\rho_{i *}\left(D_{i}^{\prime}\right)=\underline{d_{i}}-\left[v_{i}\right]$ and $r_{X_{i}}\left(D_{i}^{\prime}\right) \geq r_{\bar{G}_{i}}\left(\underline{d_{i}}-\left[v_{i}\right]\right)$. 
We remark that $X=X_{1} \cup X_{2}$ and that $X_{1} \cap X_{2}$ consists of the node of $X$ corresponding to the edge $e$. Let $p$ denote this node. Since $p$ is a smooth point on $X_{i}$, the Weil divisor $[p]$ is regarded as a Cartier divisor on $X_{i}$. We set

$$
D_{i}=D_{i}^{\prime}+[p] \in \operatorname{Div}\left(X_{i}\right) .
$$

By Remark 3.4, there exists a Cartier divisor $D$ on $X$ such that $\left.D\right|_{X_{i}}$ is linearly equivalent to $D_{i}$ on $X_{i}$. Then we have $\rho_{*}(D)=\underline{d}$ as in Case 1 .

For $i=1,2$, we set

$$
\varepsilon_{i}= \begin{cases}1 & \text { if } p \in \operatorname{Bs}\left(D_{i}\right), \\ 0 & \text { if } p \notin \operatorname{Bs}\left(D_{i}\right),\end{cases}
$$

so that $r_{X_{i}}\left(D_{i}^{\prime}\right)=r_{X}\left(D_{i}\right)-\left(1-\varepsilon_{i}\right)$. Then it follows from Lemma 3.3 that

$$
\begin{aligned}
r_{X}(D) & =r_{X_{1}}\left(\left.D\right|_{X_{1}}\right)+r_{X_{2}}\left(\left.D\right|_{X_{2}}\right)+\varepsilon_{1} \varepsilon_{2} \\
& =r_{X_{1}}\left(D_{1}\right)+r_{X_{2}}\left(D_{2}\right)+\varepsilon_{1} \varepsilon_{2} \\
& =r_{X_{1}}\left(D_{1}^{\prime}\right)+r_{X_{2}}\left(D_{2}^{\prime}\right)+\left(1-\varepsilon_{1}\right)+\left(1-\varepsilon_{2}\right)+\varepsilon_{1} \varepsilon_{2} \\
& \geq r_{\bar{G}_{1}}\left(\underline{d_{1}}-\left[v_{1}\right]\right)+r_{\bar{G}_{2}}\left(\underline{d_{2}}-\left[v_{2}\right]\right)+1+\left(1-\varepsilon_{1}\right)\left(1-\varepsilon_{2}\right) \\
& \geq r_{\bar{G}_{1}}\left(\underline{d_{1}}-\left[v_{1}\right]\right)+r_{\bar{G}_{2}}\left(\underline{d_{2}}-\left[v_{2}\right]\right)+1 .
\end{aligned}
$$

This gives the desired properties in the remaining case, thus completing the proof.

Next, we reinterpret Proposition 1.3.

\section{LEMMA 4.2}

In the setting of Proposition 1.3, we have

$$
r_{\bar{G}}(\underline{d}) \leq\left\{\begin{array}{c}
r_{\bar{G}_{1}}\left(\underline{d_{1}}-\left[v_{1}\right]\right)+r_{\bar{G}_{2}}\left(\underline{d_{2}}-\left[v_{2}\right]\right)+2 \\
\text { if } v_{i} \notin \operatorname{Bs}\left(\left|\underline{d_{i}}\right|^{\bullet}\right) \text { for each } i=1,2, \\
r_{\bar{G}_{1}}\left(\underline{d_{1}}-\left[v_{1}\right]\right)+r_{\bar{G}_{2}}\left(\underline{d_{2}}-\left[v_{2}\right]\right)+1 \\
\text { otherwise. }
\end{array}\right.
$$

Proof

First we consider the case where $v_{i} \notin \operatorname{Bs}\left(\left|\underline{d}_{i}\right|^{\bullet}\right)$ for each $i=1,2$. Then, since $r_{\bar{G}_{i}}\left(\underline{d_{i}}-\left[v_{i}\right]\right)=r_{\bar{G}_{i}}\left(\underline{d_{i}}\right)-1$, it follows from Proposition 1.3 that

$$
r_{\bar{G}}(\underline{d}) \leq r_{\bar{G}_{1}}\left(\underline{d_{1}}\right)+r_{\bar{G}_{2}}\left(\underline{d_{2}}\right)=r_{\bar{G}_{1}}\left(\underline{d_{1}}-\left[v_{1}\right]\right)+r_{\bar{G}_{2}}\left(\underline{d_{2}}-\left[v_{2}\right]\right)+2 .
$$

This shows the inequality (4.2) in this case.

Next consider the case where $v_{1} \in \operatorname{Bs}\left(\left|\underline{d_{1}}\right|^{\bullet}\right)$ and $v_{2} \notin \operatorname{Bs}\left(\left|\underline{d_{2}}\right|^{\bullet}\right)$. (The case of $v_{1} \notin \operatorname{Bs}\left(\left|\underline{d_{1}}\right|^{\bullet}\right)$ and $v_{2} \in \operatorname{Bs}\left(\left|\underline{d_{2}}\right|^{\bullet}\right)$ is shown in the same way.) Then we have $r_{\bar{G}_{1}}\left(\underline{d_{1}}-\left[v_{1}\right]\right)=r_{\bar{G}_{1}}\left(\underline{d_{1}}\right)$, and $r_{\bar{G}_{2}}\left(\underline{d_{2}}-\left[v_{2}\right]\right)=r_{\bar{G}_{2}}\left(\underline{d_{2}}\right)-1$. It follows from Proposition 1.3 that

$$
r_{\bar{G}}(\underline{d}) \leq r_{\bar{G}_{1}}\left(\underline{d_{1}}\right)+r_{\bar{G}_{2}}\left(\underline{d_{2}}\right)=r_{\bar{G}_{1}}\left(\underline{d_{1}}-\left[v_{1}\right]\right)+r_{\bar{G}_{2}}\left(\underline{d_{2}}-\left[v_{2}\right]\right)+1,
$$


which shows (4.2) in this case.

Finally, consider the case where $v_{i} \in \operatorname{Bs}\left(\left|\underline{d}_{i}\right|^{\bullet}\right)$ for each $i=1,2$. This means that $r_{\bar{G}_{i}}\left(\underline{d_{i}}-\left[v_{i}\right]\right)=r_{\bar{G}_{i}}\left(\underline{d_{i}}\right)$, and Proposition 1.3 gives

$$
r_{\bar{G}}(\underline{d}) \leq r_{\bar{G}_{1}}\left(\underline{d_{1}}\right)+r_{\bar{G}_{2}}\left(\underline{d_{2}}\right)+1=r_{\bar{G}_{1}}\left(\underline{d_{1}}-\left[v_{1}\right]\right)+r_{\bar{G}_{2}}\left(\underline{d_{2}}-\left[v_{2}\right]\right)+1 .
$$

Thus, we obtain (4.2).

To prove Theorem 1.1, we consider the following condition for a vertex-weighted graph $\bar{G}$.

(FS) There exists a nodal curve $X$ with dual graph $\bar{G}$ such that, for any $\underline{d} \in \operatorname{Div}(G)$, there exists a Cartier divisor $D$ on $X$ such that $\rho_{*}(D)=\underline{d}$ and $r_{X}(D) \geq r_{\bar{G}}(\underline{d})$, where $\rho_{*}: \operatorname{Div}(X) \rightarrow \operatorname{Div}(G)$ is the map defined in (2.1).

We note that if a vertex-weighted graph $\bar{G}$ satisfies the condition (FS), then we have $r_{\bar{G}}^{\mathrm{alg}}(\delta) \geq r_{\bar{G}}(\delta)$ for any divisor class $\delta \in \operatorname{Pic}(G)$. Indeed, let $\delta$ be any divisor class of $G$. We take the nodal curve $X$ as in the condition (FS). Then for any representative $\underline{d} \in \operatorname{Div}(G)$ of $\delta$, we take a Cartier divisor $D$ on $X$ as in (FS). With $X$ as above, we obtain $\min _{\underline{d} \in \delta}\left\{\max _{L \in \operatorname{Pic} \underline{d}(X)}\left\{r_{X}(L)\right\}\right\} \geq r_{\bar{G}}(\delta)$. Thus, we get $r_{\bar{G}}^{\mathrm{alg}}(\delta) \geq r_{\bar{G}}(\delta)$.

\section{Proof of Theorem 1.1}

Let $\bar{G}$ be a hyperelliptic vertex-weighted graph. We will show that $\bar{G}$ satisfies the condition (FS) by induction on the number of bridges. As is explained above, we will then have the desired inequality $r_{\bar{G}}^{\text {alg }}(\delta) \geq r_{\bar{G}}(\delta)$ for any divisor class $\delta$ on $G$.

If $G$ has no bridges, then [14, Proposition 1.5 and its proof and Theorem 8.2] tells us that $\bar{G}$ satisfies the condition (FS). (More generally, if there are at most $(2 \omega(v)+2)$ positive-type bridges emanating from each vertex $v \in V(G)$, then $\bar{G}$ satisfies the condition (FS) (see [14]).) Also, a vertex-weighted graph of genus at most 1 satisfies the condition (FS) (cf. [14, Proposition 1.5 and its proof and Proposition 7.5]).

Now we consider the general case, and suppose that $\bar{G}$ has a bridge. Let $G_{1}$ and $G_{2}$ be the connected components of $G \backslash\{e\}$, and set $\bar{G}_{i}=\left(G_{i},\left.\omega\right|_{V\left(G_{i}\right)}\right)$ for $i=1,2$. Then we find that $\bar{G}_{i}$ is a hyperelliptic or $g\left(\bar{G}_{i}\right) \leq 1$ (see [6, Section 5.2] or [14, Lemma 3.4]).

By the induction on the number of bridges, we may and do assume that $\bar{G}_{i}$ satisfies the condition (FS) for each $i=1,2$. Thus, there exists a nodal curve $X_{i}$ such that, for any $\underline{e_{i}} \in \operatorname{Div}\left(G_{i}\right)$, there exists a Cartier divisor $E_{i}$ on $X_{i}$ satisfying $\rho_{i *}\left(E_{i}\right)=\underline{e_{i}}$ and $\bar{r}_{X_{i}}\left(E_{i}\right) \geq r_{\bar{G}_{i}}\left(\underline{e_{i}}\right)$, where $\rho_{i *}: \operatorname{Div}\left(X_{i}\right) \rightarrow \operatorname{Div}\left(G_{i}\right)$ is the map defined in (2.1).

Let $p_{i}$ be a smooth point of $X_{i}$ for each $i=1,2$. Then we patch $X_{1}$ and $X_{2}$ by $p_{1}=p_{2}(=: p)$ to obtain a nodal curve $X$ such that $X=X_{1} \cup X_{2}$ and $X_{1} \cap X_{2}=\{p\}$. Here we take each $p_{i}$ so that $X=X_{1} \cup X_{2}$ is a nodal curve with dual graph $\bar{G}$ and so that each $G_{i}$ is the subgraph of $G$ corresponding to the component $X_{i}$. Let $\rho_{*}: \operatorname{Div}(X) \rightarrow \operatorname{Div}(G)$ be the map defined in (2.1). 
We prove that, with this $X, \bar{G}$ satisfies the condition (FS). Indeed, let $\underline{d}$ be any divisor on $G$. For $i=1,2$, let $\underline{d}_{i}$ be the restriction of $\underline{d}$ to $G_{i}$. By Lemma 4.1, there exists a Cartier divisor $D$ on $X$ satisfying $\rho_{*}(D)=\underline{d}$ and $r_{X}(D) \geq \begin{cases}r_{\bar{G}_{1}}\left(\underline{d_{1}}-\left[v_{1}\right]\right)+r_{\bar{G}_{2}}\left(\underline{d_{2}}-\left[v_{2}\right]\right)+2 & \text { if } v_{i} \notin \operatorname{Bs}\left(\left|\underline{d_{i}}\right|^{\bullet}\right) \text { for each } i=1,2, \\ r_{\bar{G}_{1}}\left(\underline{d_{1}}-\left[v_{1}\right]\right)+r_{\bar{G}_{2}}\left(\underline{d_{2}}-\left[v_{2}\right]\right)+1 & \text { otherwise. }\end{cases}$

By Lemma 4.2, the right-hand side is at least $r_{\bar{G}}(\underline{d})$. Thus, we obtain $r_{X}(D) \geq$ $r_{\bar{G}}(\underline{d})$, which shows that $\bar{G}$ satisfies the condition (FS).

\section{Rank of divisors on graphs and curves of genus 3}

In this section, we prove Proposition 1.4 and then Theorem 1.2. Let $R$ be a complete discrete valuation ring with fractional field $\mathbb{K}$ and residue field $k$. Let $\mathscr{X}$ be a regular, generically smooth, semistable $R$-curve. Let $\bar{G}=(G, \omega)$ be the reduction graph of $\mathscr{X}$. Let $\widetilde{\rho}_{*}: \operatorname{Div}\left(\mathscr{X}_{\mathbb{K}}\right) \rightarrow \operatorname{Div}(G)$ be the specialization map defined in (2.2).

We begin with the proof of Proposition 1.4.

\section{Proof of Proposition 1.4}

Recall that $\bar{G}=(G, \omega)$ is a nonhyperelliptic graph of genus 3, and recall that $\mathscr{X}$ is a regular, generically smooth, semistable $R$-curve $\mathscr{X}$ with reduction graph $\bar{G}$.

First we claim that if $\operatorname{deg}(\underline{d}) \leq 2$, then $r_{\bar{G}}(\underline{d}) \leq 0$. Indeed, to argue by contradiction, suppose that $r_{\bar{G}}(\underline{d}) \geq 1$. Since $r_{\bar{G}}(\underline{d}) \leq \operatorname{deg}(\underline{d})$, this means $(1) \operatorname{deg}(\underline{d})=1$ and $r_{\bar{G}}(\underline{d})=1 ;(2) \operatorname{deg}(\underline{d})=2$ and $r_{\bar{G}}(\underline{d})=1$; or $(3) \operatorname{deg}(\underline{d})=2$ and $r_{\bar{G}}(\underline{d})=2$. In (1), the existence of $\underline{d}$ forces $\bar{G}^{\bullet}$ to be a tree, which is a contradiction. In (2), $\bar{G}$ is hyperelliptic, which is excluded at the beginning. In (3), there exists a vertex $v$ of $\bar{G}^{\bullet}$ such that $\left.r_{\bar{G}} \bullet \underline{d}-[v]\right)=1$ and $\operatorname{deg}(\underline{d}-[v])=1$, where we regard $\underline{d} \in \operatorname{Div}\left(\bar{G}^{\bullet}\right)$ via the natural embedding $\operatorname{Div}(G) \subseteq \operatorname{Div}\left(\bar{G}^{\bullet}\right)$ as before. The existence of the divisor $\underline{d}-[v]$ forces $\bar{G}^{\bullet}$ to be a tree, which is a contradiction. Hence we obtain the claim.

Case 1. Suppose that $r_{\bar{G}}(\underline{d})=-1$. By the surjectivity of the homomorphism (2.4), there exists $\widetilde{D} \in \operatorname{Div}\left(\mathscr{X}_{\mathbb{K}}\right)$ with $\widetilde{\rho}_{*}(\widetilde{D})=\underline{d}$. Then the specialization lemma (Theorem 2.7) tells us that $-1=r_{\bar{G}}(\underline{d}) \geq r_{\mathscr{X}_{\mathbb{K}}}(\widetilde{D})$. It follows that $r_{\mathscr{X}_{\mathbb{K}}}(\widetilde{D})=-1$. Thus the equality $r_{\mathscr{X}_{\mathbb{K}}}(\widetilde{D})=r_{\bar{G}}(\underline{d})$ holds.

Case 2. Suppose that $r_{\bar{G}}(\underline{d})=0$. Then it follows from Lemma 3.2 that there exists an effective divisor $\underline{e} \in \operatorname{Div}(G)$ such that $\underline{e}$ is linearly equivalent to $\underline{d}$ in $G$. Since the homomorphism (2.4) induces a surjective map between the sets of effective divisors, there exists an effective divisor $\widetilde{E} \in \operatorname{Div}\left(\mathscr{X}_{\mathbb{K}}\right)$ with $\widetilde{\rho}_{*}(\widetilde{E})=\underline{e}$. Now we use Raynaud's theorem (Theorem A.1 below) as in the proof of [14, Theorem 1.5]. It follows that there exists a principal divisor $\tilde{N} \in \operatorname{Div}\left(\mathscr{X}_{\mathbb{K}}\right)$ such that $\widetilde{\rho}_{*}(\widetilde{N})=\underline{d}-\underline{e}$. We set $\widetilde{D}=\widetilde{E}+\widetilde{N}$. Then $\rho_{*}(\widetilde{D})=\underline{d}$. Since $\widetilde{D}$ is linearly equivalent to $\widetilde{\widetilde{E}}$ on $\mathscr{X}_{\mathbb{K}}$, we have $r_{\mathscr{X}_{\mathbb{K}}}(\widetilde{D})=r_{\mathscr{X}_{\mathbb{K}}}(\widetilde{E}) \geq 0$. Since

$$
0=r_{\bar{G}}(\underline{d})=r_{\bar{G}}(\underline{e}) \geq r_{\mathscr{X}_{\mathbb{K}}}(\widetilde{E})
$$


by the specialization lemma (Theorem 2.7 ), we obtain the equality $r_{\mathscr{X}_{\mathbb{K}}}(\widetilde{D})=$ $r_{\bar{G}}(\underline{d})(=0)$.

Case 3. Suppose that $r_{\bar{G}}(\underline{d}) \geq 1$. By the above claim, we have $\operatorname{deg}(\underline{d}) \geq 3$. We put $\underline{d^{\prime}}:=K_{\bar{G}}-\underline{d} \in \operatorname{Div}(G)$. Then $\operatorname{deg}\left(\underline{d^{\prime}}\right)=4-\operatorname{deg}(\underline{d}) \leq 1$. Thus, $r_{\bar{G}}\left(\underline{d^{\prime}}\right) \leq$ $\operatorname{deg}\left(\underline{d^{\prime}}\right) \leq 1$. Since $\bar{G}^{\bullet}$ is not a tree, we have $r_{\bar{G}}\left(\underline{d^{\prime}}\right) \neq 1$. It follows that $r_{\bar{G}}\left(\underline{d}^{\prime}\right) \leq 0$. By Cases 1 and 2, there exists a divisor $\widetilde{D^{\prime}} \in \operatorname{Div}\left(\mathscr{X}_{\mathbb{K}}\right)$ such that $\widetilde{\rho}_{*}\left(\widetilde{D^{\prime}}\right)=\underline{d^{\prime}}$ and $r_{\mathscr{X}_{\mathbb{K}}}\left(\widetilde{D^{\prime}}\right)=r_{\bar{G}}\left(\underline{d^{\prime}}\right)$.

By [4, Remark 4.18 and Remark 4.21], there exists a canonical divisor $K_{\mathscr{X}_{\mathbb{K}}}$ of $\mathscr{X}_{\mathbb{K}}$ such that $\widetilde{\rho}_{*}\left(K_{\mathscr{X}_{\mathbb{K}}}\right)=K_{\bar{G}}$. We set $\widetilde{D}:=K_{\mathscr{X}_{\mathbb{K}}}-\widetilde{D^{\prime}}$. Then we have $\widetilde{\rho}_{*}(\widetilde{D})=$ $K_{\bar{G}}-\underline{d^{\prime}}=\underline{d}$. Further, the Riemann-Roch formulae on $\mathscr{X}_{\mathbb{K}}$ and $\bar{G}$ (cf. [2, Theorem 3.8]) give

$$
r_{\mathscr{X}_{\mathbb{K}}}(\widetilde{D})=-2+\operatorname{deg}(\widetilde{D})+r_{\mathscr{X}_{\mathbb{K}}}\left(\widetilde{D^{\prime}}\right)=-2+\operatorname{deg}(\underline{d})+r_{\bar{G}}\left(\underline{d^{\prime}}\right)=r_{\bar{G}}(\underline{d}) .
$$

Thus, we obtain Proposition 1.4.

\section{Proof of Theorem 1.2}

The proof goes in the same way as in [14]; Theorem 1.2 will be deduced from Proposition 1.4.

Recall that $k$ is a fixed algebraically closed field. We take a complete discrete valuation ring $R$ with residue field $k$. For example, we may take $R$ as the ring of formal power series $k \llbracket t \rrbracket$ over $k$. Let $\mathbb{K}$ be the fractional field of $R$. We take a regular, generically smooth, semistable $R$-curve $\mathscr{X}$ with reduction graph $\bar{G}$. We note that such $\mathscr{X}$ always exists (see [4, Theorem B.2]).

Let $\mathscr{X}_{\mathbb{K}}$ denote the generic fiber of $\mathscr{X}$, and let $X$ denote the special fiber of $\mathscr{X}$. For $\underline{d} \in \operatorname{Div}(G)$, Proposition 1.4 shows that there exists a divisor $\widetilde{D} \in$ $\operatorname{Div}\left(\mathscr{X}_{\mathbb{K}}\right)$ such that $\widetilde{\rho}_{*}(\widetilde{D})=\underline{d}$ and $r_{\bar{G}}(\underline{d})=r_{\mathscr{X}_{\mathbb{K}}}(\widetilde{D})$. Let $\widetilde{\mathscr{D}}$ be the Zariski closure of $\widetilde{D}$ in $\mathscr{X}$. We denote by $\mathcal{O}_{\mathscr{X}}(\widetilde{\mathscr{D}})$ the invertible sheaf on $\mathscr{X}$ associated to $\widetilde{\mathscr{D}}$. Let $D \in \operatorname{Div}(X)$ be a divisor on $X$ such that the associated invertible sheaf $\mathcal{O}_{X}(D)$ is isomorphic to $\left.\mathcal{O}_{\mathscr{X}}(\widetilde{\mathscr{D}})\right|_{X}$. By the upper-semicontinuity of the cohomology, we have $r_{X}(D) \geq r_{\mathscr{X}_{\mathbb{K}}}(\widetilde{D})$. Hence, $r_{X}(D) \geq r_{\bar{G}}(\underline{d})$. Also, by $(2.3)$, we have $\rho_{*}(D)=\underline{d}$. If follows that $\bar{G}$ satisfies the condition (FS) in Section 4 , and we get $r_{\bar{G}}^{\text {alg }}(\delta) \geq$ $r_{\bar{G}}(\delta)$ for any divisor class $\delta \in \operatorname{Pic}(G)$.

In the rest of this section, we will show a metric graph version of Proposition 1.4. Let $\mathscr{X}$ be a regular, generically smooth, semistable $R$-curve with reduction graph $\bar{G}=(G, \omega)$. Let $\Gamma$ be the metric graph associated to $G$, where each edge of $G$ is assigned length one. Let $\Gamma_{\mathbb{Q}}$ be the set of points of $\Gamma$ whose distance from every vertex of $G$ is rational.

We follow the arguments in $\left[4\right.$, Section 2.3]. Let $\mathbb{K}^{\prime} / \mathbb{K}$ be a finite extension. Let $R^{\prime}$ be the ring of integers of $\mathbb{K}^{\prime}$. Then $R^{\prime}$ is a complete discrete valuation ring with residue field $k$. Let $\mathscr{X}^{\prime}$ be the minimal resolution of $\mathscr{X} \times_{\operatorname{Spec}(R)} \operatorname{Spec}\left(R^{\prime}\right)$. Then $\mathscr{X}^{\prime}$ is a regular, generically smooth, semistable $R^{\prime}$-curve with generic fiber $\mathscr{X} \times \operatorname{Spec(\mathbb {K})} \operatorname{Spec}\left(\mathbb{K}^{\prime}\right)$. Let $e\left(\mathbb{K}^{\prime} / \mathbb{K}\right)$ be the ramification index of $\mathbb{K}^{\prime} / \mathbb{K}$. The dual graph $\bar{G}^{\prime}=\left(G^{\prime}, \omega^{\prime}\right)$ of the special fiber of $\mathscr{X}^{\prime}$ is the graph obtained by inserting 
$e\left(\mathbb{K}^{\prime} / \mathbb{K}\right)-1$ vertices to each edge of $G$, and $\omega^{\prime}$ is the extension of $\omega$, where $\omega^{\prime}(w)=0$ for any $w \in V\left(G^{\prime}\right) \backslash V(G)$. If we assign a length of $1 / e\left(\mathbb{K}^{\prime} / \mathbb{K}\right)$ to each edge of $G^{\prime}$, then the corresponding metric graph equals $\Gamma$. The pair $\Gamma$ with a vertex-weight function $\Gamma \rightarrow \mathbb{Z}$ (given by the zero extension of $\omega$ ) is denoted by $\bar{\Gamma}$.

Let $\overline{\mathbb{K}}$ be an algebraic closure of $\mathbb{K}$. For $\widetilde{D} \in \operatorname{Div}\left(\mathscr{X}_{\overline{\mathbb{K}}}\right)$, we take a finite extension $\mathbb{K}^{\prime} / \mathbb{K}$ such that $\widetilde{D} \in \operatorname{Div}\left(\mathscr{X}\left(\mathbb{K}^{\prime}\right)\right)$, and then we set $\widetilde{\tau}_{*}(\widetilde{D})={\widetilde{\rho^{\prime}}}^{\prime}(\widetilde{D})$, where ${\widetilde{\rho^{\prime}}}_{*}$ is the specialization map for $\mathscr{X}^{\prime}$. This gives rise to the specialization map

$$
\widetilde{\tau}_{*}: \operatorname{Div}\left(\mathscr{X}_{\overline{\mathbb{K}}}\right) \rightarrow \operatorname{Div}\left(\Gamma_{\mathbb{Q}}\right)
$$

(This map is denoted by $\tau_{*}$ in [14]. Here we write $\widetilde{\tau}_{*}$ instead because of the compatibility with the notation $\widetilde{\rho}_{*}$ (cf. Remark 2.6).) We remark that the metric graph $\Gamma$ can be seen as the Berkovich skeleton $S(\mathscr{X})$ and that the specialization map is generalized to the strong retraction map from the Berkovich space $\left(\mathscr{X}_{\overline{\mathbb{K}}}\right)^{\text {an }}$ to $S(\mathscr{X})$ (see [7] for details).

For each $\underline{d} \in \operatorname{Div}\left(\Gamma_{\mathbb{Q}}\right)$, we take a graph $\bar{G}^{\prime}=\left(G^{\prime}, \omega^{\prime}\right)$ with $\operatorname{Supp}(\underline{d}) \subset V\left(G^{\prime}\right)$, and define $r_{\bar{\Gamma}}(\underline{d}):=r_{\bar{G}^{\prime}}(\underline{d})$, which does not depend on the choice of $\bar{G}^{\prime}$ by $[2$, Section 1]. By Amini-Caporaso's specialization lemma (Theorem 2.7), we have $r_{\bar{G}^{\prime}}\left(\rho_{*}^{\prime}(\widetilde{D})\right) \geq r_{\mathscr{X}_{\mathbb{K}^{\prime}}}(\widetilde{D})$ for any $\widetilde{D} \in \operatorname{Div}\left(\mathscr{X}_{\mathbb{K}^{\prime}}\right)$. As is mentioned in [4, Remark 2.9], if $\widetilde{D} \in \operatorname{Div}\left(\mathscr{X}_{\mathbb{K}^{\prime}}\right) \backslash \operatorname{Div}\left(\mathscr{X}\left(\mathbb{K}^{\prime}\right)\right)$, then $\widetilde{\rho}^{\prime}{ }_{*}(\widetilde{D})$ and $\widetilde{\tau}_{*}(\widetilde{D})$ may be different, but $\widetilde{\rho}_{*}(\widetilde{D})$ and $\widetilde{\tau}_{*}(\widetilde{D})$ are at least linearly equivalent in $G^{\prime}$. Thus, we have the specialization lemma for vertex-weighted metric graphs: for any $\widetilde{D} \in \operatorname{Div}\left(\mathscr{X}_{\overline{\mathbb{K}}}\right)$, one has

$$
r_{\bar{\Gamma}}\left(\tau_{*}(\widetilde{D})\right) \geq r_{\mathscr{X}_{\overline{\mathbb{R}}}}(\widetilde{D}) .
$$

Also for metric graphs, we have Raynaud's theorem, which asserts the surjectivity of the map $\left.\widetilde{\tau}_{*}\right|_{\operatorname{Prin}\left(\mathscr{X}_{\overline{\mathbb{K}}}\right)}: \operatorname{Prin}\left(\mathscr{X}_{\overline{\mathbb{K}}}\right) \rightarrow \operatorname{Prin}\left(\Gamma_{\mathbb{Q}}\right)$ (see [4, Corollary A.9]). Then, by the same argument as in the proof of Proposition 1.4, we obtain the following proposition.

\section{PROPOSITION 5.1}

Let $R$ be a complete discrete valuation ring with fractional field $\mathbb{K}$ and residue field $k$. Let $\bar{G}=(G, \omega)$ be a nonhyperelliptic graph of genus 3 , and let $\Gamma$ be the metric graph associated to $G$, where each edge of $G$ is assigned length one. Let $\mathscr{X}$ be a regular, generically smooth, semistable $R$-curve with reduction graph $\bar{G}$. Then the following condition $(C)$ holds.

(C) For any $\underline{d} \in \operatorname{Div}\left(\Gamma_{\mathbb{Q}}\right)$, there exists a divisor $\widetilde{D} \in \operatorname{Div}\left(\mathscr{X}_{\mathbb{\mathbb { K }}}\right)$ such that $\widetilde{\tau}_{*}(\widetilde{D})=\underline{d}$ and $r_{\bar{\Gamma}}(\underline{d})=r_{\mathscr{X}_{\overline{\mathbb{K}}}}(\widetilde{D})$.

\section{REMARK 5.2}

Let $R$ be a complete discrete valuation ring with fractional field $\mathbb{K}$ and residue field $k$. Let $\bar{G}=(G, \omega)$ be a vertex-weighted graph. In [14], we have asked under what condition on $\bar{G}$ there exists a regular, generically smooth, semistable $R$ curve with reduction graph $\bar{G}$ that satisfies the conditions (F) and (C) in Propositions 1.4 and 5.1, respectively. In [14], when $\operatorname{char}(k) \neq 2$, we have completely 
answered this question for hyperelliptic graphs: a hyperelliptic graph $\bar{G}=(G, \omega)$ satisfies the conditions (F) and (C) if and only if every vertex $v$ of $G$ has at most $2 \omega(v)+2$ positive-type bridges emanating from it. In this paper, we answer this question for nonhyperelliptic graphs of genus 3: every nonhyperelliptic graph of genus 3 satisfies (F) and (C). It is then natural to ask this question for nonhyperelliptic graphs of genus 4 . In this case, the arguments in the proof of Proposition 1.4 show the existence of a desired lift $\widetilde{D}$ of $\underline{d}$ except for divisors $\underline{d}$ with $\operatorname{deg}(\underline{d})=3$ and $r_{\bar{G}}(\underline{d})=1$.

\section{REMARK 5.3}

Assume that $\operatorname{char}(k) \neq 2$. Let $\bar{G}=(G, \omega)$ be a vertex-weighted graph. Let $e_{1}$, $\ldots, e_{r}$ be the set of bridges of $G$, and we write $G \backslash\left\{e_{1}, \ldots, e_{r}\right\}=G_{1} \cup \cdots \cup G_{r+1}$ as the disjoint union of connected finite graphs. We set $\bar{G}_{i}=\left(G_{i},\left.\omega\right|_{G_{i}}\right)$. The proofs of Theorems 1.1 and 1.2 show that hyperelliptic graphs and graphs of genus at most 3 satisfy the condition (FS). It follows from the proof of Theorem 1.1 that if each $\bar{G}_{i}$ is hyperelliptic or of genus at most 3 , then $\bar{G}$ satisfies the condition (FS), and thus we have $r_{\bar{G}}^{\operatorname{alg}}(\delta) \geq r_{\bar{G}}(\delta)$ for any divisor class $\delta \in \operatorname{Pic}(G)$.

\section{Appendix: Raynaud's theorem}

The purpose of this appendix is to show that, for a finite graph with loops, the specialization map between principal divisors is still surjective. Our proof of the surjectivity will be given by reducing to the case of loopless finite graphs. The surjectivity in the loopless case is shown in Baker [4].

In [4], the surjectivity of the specialization map (in the loopless case) is attributed to Raynaud because this surjectivity follows from reinterpretation of Raynaud's results in [16] (see [4, Appendix A]). In this paper, we also call Theorem A.1, which asserts the surjectivity, Raynaud's theorem.

Let $k$ be an algebraically closed field as before. Let $R$ be a complete valuation ring with residue field $k$. Let $\mathbb{K}$ be the fractional field of $R$. Let $\mathscr{X} \rightarrow \operatorname{Spec}(R)$ be a regular, generically smooth, semistable $R$-curve. We write $X$ for the special fiber of $\mathscr{X}$, and $\bar{G}=(G, \omega)$ for the dual graph of $X$. Let $\widetilde{\rho}_{*}: \operatorname{Div}\left(\mathscr{X}_{\mathbb{K}}\right) \rightarrow \operatorname{Div}(G)$ be the specialization map defined in (2.2).

\section{THEOREM A.1}

The specialization map between principal divisors is surjective. Namely, $\left.\widetilde{\rho}_{*}\right|_{\operatorname{Prin}\left(\mathscr{X}_{\mathbb{K}}\right)}: \operatorname{Prin}\left(\mathscr{X}_{\mathbb{K}}\right) \rightarrow \operatorname{Prin}(G)$ is surjective.

\section{Proof}

We put $p:=\operatorname{char}(k) \geq 0$. When $G$ is loopless, the assertion is exactly [4, Corollary A.8]. We will reduce the general case to the loopless case.

Let $d$ be an integer with $d \geq 2$. When $p>0$, we require that $(d, p)=1$. We fix a finite Galois extension $\mathbb{K}^{\prime}$ of $\mathbb{K}$ of degree $d$. (For example, we may take $\mathbb{K}^{\prime}=\mathbb{K}(\sqrt[d]{\pi})$, where $\pi \in R$ is a uniformizer of $R$.) Since $k$ is algebraically closed 
and $\mathbb{K}^{\prime} / \mathbb{K}$ is a Galois extension of degree $d$, the ramification index $e\left(\mathbb{K}^{\prime} / \mathbb{K}\right)$ equals d. We denote by $R^{\prime}$ the ring of integers of $\mathbb{K}^{\prime}$.

Let $\mathscr{X}^{\prime}$ be the minimal resolution of $\mathscr{X} \times_{\operatorname{Spec}(R)} \operatorname{Spec}\left(R^{\prime}\right)$. Let $\nu: \mathscr{X}^{\prime} \rightarrow \mathscr{X}$ be the natural map. By slight abuse of notation, we denote the restriction of $\nu$ to the generic fibers by the same notation $\nu$. Let $X^{\prime}$ be the special fiber of $\mathscr{X}^{\prime}$. Let $G^{\prime}$ be dual graph of $X^{\prime}$, and let $\widetilde{\rho_{*}^{\prime}}: \operatorname{Div}\left(\mathscr{X}_{\mathbb{K}^{\prime}}\right) \rightarrow \operatorname{Div}\left(G^{\prime}\right)$ be the specialization map with respect to $\mathscr{X}^{\prime}$. Since $G^{\prime}$ is the graph obtained by inserting $(d-1)$ vertices to each edge of $G$, we have a natural embedding $V(G) \subseteq V\left(G^{\prime}\right)$ and also $\operatorname{Div}(X) \subseteq \operatorname{Div}\left(X^{\prime}\right)$.

\section{CLAIM A.1.1}

Let $\widetilde{D} \in \operatorname{Div}\left(\mathscr{X}_{\mathbb{K}}\right)$ such that $\widetilde{\rho_{*}^{\prime}}\left(\nu^{*}(\widetilde{D})\right) \in \operatorname{Div}(G)$. Then $\widetilde{\rho_{*}^{\prime}}\left(\nu^{*}(\widetilde{D})\right)=\widetilde{\rho}_{*}(\widetilde{D})$.

Indeed, we take any $v \in V(G)$. Let $C_{v}$ be the irreducible component of $X$ corresponding to $v$, and let $C_{v}^{\prime}$ be the irreducible component of $X^{\prime}$ with $\nu\left(C_{v}^{\prime}\right)=C_{v}$. Let $\mathscr{D} \in \operatorname{Div}(\mathscr{X})$ and $\mathscr{D}^{\prime} \in \operatorname{Div}\left(\mathscr{X}^{\prime}\right)$ be the Zariski closures of $\widetilde{D}$ and $\nu^{*}(\widetilde{D})$, respectively. We have $\widetilde{\rho}_{*}(\widetilde{D})(v)=\left(C_{v} \cdot \mathscr{D}\right)$ and $\widetilde{\rho_{*}^{\prime}}\left(\nu^{*}(\widetilde{D})\right)(v)=\left(C_{v}^{\prime} \cdot \mathscr{D}^{\prime}\right)$.

Since $\nu_{*}\left(\mathscr{D}^{\prime}\right)=d \mathscr{D}$, we have $d\left(C_{v} \cdot \mathscr{D}\right)=\left(\nu^{*}\left(C_{v}\right) \cdot \mathscr{D}^{\prime}\right)$ by the projection formula. By the assumption that $\widetilde{\rho_{*}^{\prime}}\left(\nu^{*}(D)\right) \in \operatorname{Div}(G)$, we have $\left(E^{\prime} \cdot \mathscr{D}^{\prime}\right)=0$ for any exceptional prime divisor $E^{\prime}$ for $\nu$. Since $\nu^{*}\left(C_{v}\right)-d C_{v}^{\prime}$ is a linear combination of exceptional divisors, it follows that $\left(\nu^{*}\left(C_{v}\right) \cdot \mathscr{D}^{\prime}\right)=d\left(C_{v}^{\prime} \cdot \mathscr{D}^{\prime}\right)$.

Then

$$
\widetilde{\rho}_{*}(\widetilde{D})(v)=\left(C_{v} \cdot \mathscr{D}\right)=\frac{\left(\nu^{*}\left(C_{v}\right) \cdot \mathscr{D}^{\prime}\right)}{d}=\left(C_{v}^{\prime} \cdot \mathscr{D}^{\prime}\right)=\widetilde{\rho_{*}^{\prime}}\left(\nu^{*}(\widetilde{D})\right)(v) .
$$

Since $\widetilde{\rho_{*}^{\prime}}\left(\nu^{*}(\widetilde{D})\right) \in \operatorname{Div}(G)$ and $v \in V(G)$ is arbitrary, we obtain Claim A.1.1.

Let $\sigma_{1}, \ldots, \sigma_{d}$ be the elements of $\operatorname{Gal}\left(\mathbb{K}^{\prime} / \mathbb{K}\right)$. Each $\sigma_{i}$ induces an automorphism $\sigma_{i}^{*}: \operatorname{Spec}\left(R^{\prime}\right) \rightarrow \operatorname{Spec}\left(R^{\prime}\right)$ and an automorphism $\varphi_{i}: \mathscr{X}^{\prime} \rightarrow \mathscr{X}^{\prime}$ over $R$ (induced from the Cartesian product). Let $\varphi_{i}^{*}: \operatorname{Div}\left(\mathscr{X}^{\prime}\right) \rightarrow \operatorname{Div}\left(\mathscr{X}^{\prime}\right)$ and $\varphi_{i}^{*}: \operatorname{Div}\left(\mathscr{X}_{\mathbb{K}^{\prime}}\right) \rightarrow \operatorname{Div}\left(\mathscr{X}_{\mathbb{K}^{\prime}}\right)$ be the induced maps.

\section{CLAIM A.1.2}

For any $\widetilde{D^{\prime}} \in \operatorname{Div}\left(\mathscr{X}_{\mathbb{K}^{\prime}}\right)$, we have $\widetilde{\rho_{*}^{\prime}}\left(\left(\varphi_{i}\right)^{*}\left(\widetilde{D^{\prime}}\right)\right)=\widetilde{\rho_{*}^{\prime}}\left(\widetilde{D^{\prime}}\right)$ for $i=1, \ldots, d$.

Indeed, since $\sigma_{i}$ induces the trivial action on the residue field $k$, the restriction of $\varphi_{i}$ to the special fiber $X^{\prime}$ is trivial. Thus, $\left(\varphi_{i}\right)_{*}\left(C^{\prime}\right)=C^{\prime}$ for any irreducible component $C^{\prime}$ of $X^{\prime}$.

We take any $\widetilde{D}^{\prime} \in \operatorname{Div}\left(\mathscr{X}_{\mathbb{K}^{\prime}}\right)$ and let $\mathscr{D}^{\prime}$ be the Zariski closure of $\widetilde{D^{\prime}}$ in $\mathscr{X}^{\prime}$. Note that $\varphi_{i}^{*}\left(\mathscr{D}^{\prime}\right)$ is the Zariski closure of $\varphi_{i}^{*}\left(\widetilde{D^{\prime}}\right)$. For any $v \in V\left(G^{\prime}\right)$, let $C_{v}^{\prime}$ be the corresponding irreducible component of $X^{\prime}$. Then

$$
\widetilde{\rho_{*}^{\prime}}\left(\varphi_{i}^{*}\left(\widetilde{D^{\prime}}\right)\right)(v)=\left(C_{v}^{\prime} \cdot \varphi_{i}^{*}\left(\mathscr{D}^{\prime}\right)\right)=\left(\left(\varphi_{i}\right)_{*}\left(C_{v}^{\prime}\right) \cdot \mathscr{D}^{\prime}\right)=\left(C_{v}^{\prime} \cdot \mathscr{D}^{\prime}\right)=\widetilde{\rho_{*}^{\prime}}\left(\widetilde{D^{\prime}}\right)(v),
$$

which shows the desired equality. We obtain Claim A.1.2.

We take any $\underline{n} \in \operatorname{Prin}(G)$. Then $\underline{n} \in \operatorname{Prin}\left(G^{\prime}\right)$. Since $G^{\prime}$ is loopless, we know that $\widetilde{\rho_{*}^{\prime}}: \operatorname{Prin}\left(\mathscr{X}_{\mathbb{K}^{\prime}}\right) \rightarrow \operatorname{Prin}\left(G^{\prime}\right)$ is surjective by $[4$, Corollary A.8]. Let $f$ be 
a nonzero rational function on $\mathscr{X}_{\mathbb{K}^{\prime}}$ such that $\widetilde{\rho_{*}^{\prime}}(\operatorname{div}(f))=\underline{n}$. We set $g^{\prime}:=$ $\varphi_{1}^{*}(f) \cdots \varphi_{d}^{*}(f)$, which is a nonzero rational function on $\mathscr{X}_{\mathbb{K}^{\prime}}$. Then $\operatorname{div}\left(g^{\prime}\right)=$ $\varphi_{1}^{*}(\operatorname{div}(f))+\cdots+\varphi_{d}^{*}(\operatorname{div}(f))$, so that Claim A.1.2 tells us that $\widetilde{\rho_{*}^{\prime}}\left(\operatorname{div}\left(g^{\prime}\right)\right)=d \underline{n}$. Since $g^{\prime}$ is a $\operatorname{Gal}\left(\mathbb{K}^{\prime} / \mathbb{K}\right)$-invariant function on $\mathscr{X}_{\mathbb{K}^{\prime}}$, it descends to a function $g$ on $\mathscr{X}_{\mathbb{K}}$. We have $\operatorname{div}\left(g^{\prime}\right)=\nu^{*}(\operatorname{div}(g))$, and thus $\widetilde{\rho_{*}^{\prime}}\left(\nu^{*}(\operatorname{div}(g))\right)=d \underline{n} \in \operatorname{Div}(G)$. By Claim A.1.1, we obtain $\widetilde{\rho}_{*}(\operatorname{div}(g))=d \underline{n}$. In conclusion, $\widetilde{L}:=\operatorname{div}(g)$ is a principal divisor on $\mathscr{X}_{\mathbb{K}}$ with $\widetilde{\rho}_{*}(\widetilde{L})=d \underline{n}$.

Let $e>2$ be another integer with $(e, d)=1$. When $p>0$, we require that $(e, p)=1$. By the above argument with $e$ in place of $d$, there exists a principal divisor $\widetilde{M} \in \operatorname{Prin}\left(\mathscr{X}_{\mathbb{K}}\right)$ with $\widetilde{\rho}_{*}(\widetilde{M})=e \underline{n}$. We take integers $\alpha$ and $\beta$ such that $\alpha d+\beta e=1$, and set $\widetilde{N}:=\alpha \widetilde{L}+\beta \widetilde{M}$. Then $\widetilde{N} \in \operatorname{Prin}\left(\mathscr{X}_{\mathbb{K}}\right)$ and $\widetilde{\rho}_{*}(\widetilde{N})=\underline{n}$. This shows the theorem.

Acknowledgments. We deeply thank Professor Lucia Caporaso for invaluable comments on this article and on [14]. We also thank the anonymous referee for carefully reading this article and giving helpful comments.

\section{References}

[1] O. Amini, M. Baker, E. Brugallé, and J. Rabinoff, Lifting harmonic morphisms, I: Metrized complexes and Berkovich skeleta, Res. Math. Sci. 2 (2015), no. 7; II: Tropical curves and metrized complexes, Algebra Number Theory 9 (2015), 267-315. MR 3375652. DOI 10.1186/s40687-014-0019-0. MR 3320845. DOI 10.2140/ant.2015.9.267.

[2] O. Amini and L. Caporaso, Riemann-Roch theory for weighted graphs and tropical curves, Adv. Math. 240 (2013), 1-23. MR 3046301.

DOI 10.1016/j.aim.2013.03.003.

[3] R. Bacher, P. de la Harpe, and T. Nagnibeda, The lattice of integral flows and the lattice of integral cuts on a finite graph, Bull. Soc. Math. France $\mathbf{1 2 5}$ (1997), 167-198. MR 1478029.

[4] M. Baker, Specialization of linear systems from curves to graphs, with an appendix by B. Conrad, Algebra Number Theory 2 (2008), 613-653. MR 2448666. DOI 10.2140/ant.2008.2.613.

[5] M. Baker and S. Norine, Riemann-Roch and Abel-Jacobi theory on a finite graph, Adv. Math. 215 (2007), 766-788. MR 2355607.

DOI 10.1016/j.aim.2007.04.012.

[6] - Harmonic morphisms and hyperelliptic graphs, Int. Math. Res. Not. IMRN 2009, no. 15, 2914-2955. MR 2525845. DOI 10.1093/imrn/rnp037.

[7] M. Baker, S. Payne, and J. Rabinoff, Nonarchimedean geometry, tropicalization, and metrics on curves, to appear in Algebr. Geom., preprint, arXiv:1104.0320v3 [math.AG]. 
[8] L. Caporaso, "Algebraic and combinatorial Brill-Noether theory" in Compact Moduli Spaces and Vector Bundles, Contemp. Math. 564, Amer. Math. Soc., Providence, 2012, 69-85. MR 2895184. DOI 10.1090/conm/564/11150.

[9] - "Rank of divisors on graphs: an algebro-geometric analysis" in A Celebration of Algebraic Geometry, Clay Math. Proc. 18, Amer. Math. Soc., Providence, 2013, 45-64. MR 3114936.

[10] , "Gonality of algebraic curves and graphs" in Algebraic and Complex Geometry, Springer Proc. Math. Stat. 71, Springer, Cham, 2014, 77-108. MR 3278571. DOI 10.1007/978-3-319-05404-9_4.

[11] L. Caporaso, Y. Len, and M. Melo, Algebraic and combinatorial rank of divisors on finite graphs, J. Math. Pures Appl. (9) 104 (2015), 227-257. MR 3365828. DOI 10.1016/j.matpur.2015.02.006.

[12] D. Cartwright, D. Jensen, and S. Payne, Lifting divisors on a generic chain of loops, Canad. Math. Bull. 58 (2015), 250-262. MR 3334919.

DOI 10.4153/CMB-2014-050-2.

[13] F. Cools, J. Draisma, S. Payne, and E. Robeva, A tropical proof of the Brill-Noether theorem, Adv. Math. 230 (2012), 759-776. MR 2914965. DOI 10.1016/j.aim.2012.02.019.

[14] S. Kawaguchi and K. Yamaki, Rank of divisors on hyperelliptic curves and graphs under specialization, Int. Math. Res. Not. IMRN 2015, no. 12, 4121-4176. MR 3356750. DOI 10.1093/imrn/rnu059.

[15] Q. Liu, Algebraic Geometry and Arithmetic Curves, Oxf. Grad. Texts Math. 6, Oxford Univ. Press, Oxford, 2002. MR 1917232.

[16] M. Raynaud, Spécialisation du foncteur de Picard, Inst. Hautes Études Sci. Publ. Math. 38 (1970), 27-76. MR 0282993.

Kawaguchi: Department of Mathematics, Graduate School of Science, Kyoto University, Kyoto, Japan; kawaguch@math.kyoto-u.ac.jp

Yamaki: Institute for Liberal Arts and Sciences, Kyoto University, Kyoto, Japan; yamaki.kazuhiko.6r@kyoto-u.ac.jp 